\title{
The First Case of Azorhizobium caulinodans Bacteremia in a Patient with Leukemia
}

\author{
Jae Hyeon Park (1), M.D. ${ }^{1,2}$, Taek Soo Kim (D), M.D. ${ }^{1,2}$, and Hyunwoong Park (D), M.D., Ph.D., ${ }^{1,3}$ \\ ${ }^{1}$ Department of Laboratory Medicine, Seoul National University College of Medicine, Seoul, Korea; ${ }^{2}$ Department of Laboratory Medicine, Seoul National \\ University Hospital, Seoul, Korea; ${ }^{3}$ Department of Laboratory Medicine, Seoul National University Seoul Metropolitan Government Boramae Medical Center, \\ Seoul, Korea
}

\section{Dear Editor,}

Azorhizobium spp. are gram-negative, rod-shaped, obligately aerobic, motile soil bacteria [1]. They are free-living microorganisms that can fix nitrogen in symbiosis with plants of the genus Sesbania [2]. Infection by Azorhizobium spp. in humans has not been reported yet. We report the first case of bacteremia caused by Azorhizobium caulinodans in a patient with AML. The Institutional Review Board of Seoul National University Hospital, Seoul, Korea approved this study (2103-179-1207) and waived the need for informed consent.

An 85-year-old male patient undergoing induction chemotherapy for AML diagnosed in July 2020 was hospitalized for additional chemotherapy in September 2020. He was in a nursing hospital before being admitted. From day 2 of hospitalization, decitabine was administered for five days. On day 2, his temperature was $37.2^{\circ} \mathrm{C}$, his heart rate was 93 beats $/ \mathrm{min}$, and his blood pressure reached $91 / 60 \mathrm{mmHg}$. A chest computed tomography scan showed destruction of the right lung after pulmonary tuberculosis and pneumonia with pleural effusion in the left lung. Hematological testing revealed a leukocyte count of $8.30 \times 10^{9} / \mathrm{L}$; the neutrophil count was $2.39 \times 10^{9} / \mathrm{L}$ with a left shift and blasts (5\%). The C-reactive protein (CRP) level was elevated to $82.6 \mathrm{mg} / \mathrm{L}$. Two pairs of peripheral blood cultures and one pair of central venous catheter (CVC) blood cultures were collected. The patient received empirical antibiotic treatment intravenously (4.5 g piperacillin/tazobactam at 6-hour intervals).

The three pairs of blood cultures were inoculated into aerobic and anaerobic blood-culture bottles and incubated in the BacT/ ALERT VIRTUO system (bioMérieux, Durham, NC, USA). Two aerobic peripheral blood cultures revealed gram-negative rods after 50 hours of incubation. Colonies on a blood agar plate appeared within 24 hours of incubation at $35^{\circ} \mathrm{C}$ and were pinpoint, round, and cream-colored (Fig. 1A). Using a GN ID card of the VITEK 2 system (bioMérieux), the gram-negative rods were identified as Bordetella bronchiseptica, with a good confidence level (91\%). The bacteria could not be identified by matrix-assisted laser desorption ionization time-of-flight mass spectrometry (MALDI-TOF MS) using a MALDI Biotyper (Bruker Daltonics, Bremen, Germany).

For accurate identification, 16S rRNA gene sequencing was performed using 27F/1492R PCR primers and 785F/907R sequencing primers [3]. The 16S rRNA sequences were searched in the GenBank database (http://www.ncbi.nlm.nih.gov/blast/) and interpreted according to the CLSI guidelines [3]. The results revealed $99.86 \%(1,396 / 1,398)$ identity with $A$. caulinodans (GenBank, NR_074185.1/NR_113675.1) and 97.93\% (1,372/1,401) identity with Xanthobacter flavus (GenBank, NR_113665.1). Phylogenetic analysis using MEGA X (http://www.megasoftware.net)
Received: October 4, 2021

Revision received: November 10, 2021

Accepted: January 17, 2022

Corresponding author: Hyunwoong Park, M.D., Ph.D. Department of Laboratory Medicine, Seoul National University Boramae Medical Center, 20 Borama-ro 5-gil, Dongjak-gu, Seoul 07061 Korea Tel: +82-2-870-2604, Fax: +82-2-870-2630

E-mail: hwparkbrmh@naver.com

\section{(c) (i) (8)}

(c) Korean Society for Laboratory Medicine

This is an Open Access article distributed under the terms of the Creative Commons Attribution Non-Commercial License (https://creativecommons.org/licenses/by-nc/4.0) which permits unrestricted non-commercial use, distribution, and reproduction in any medium, provided the original work is properly cited. 

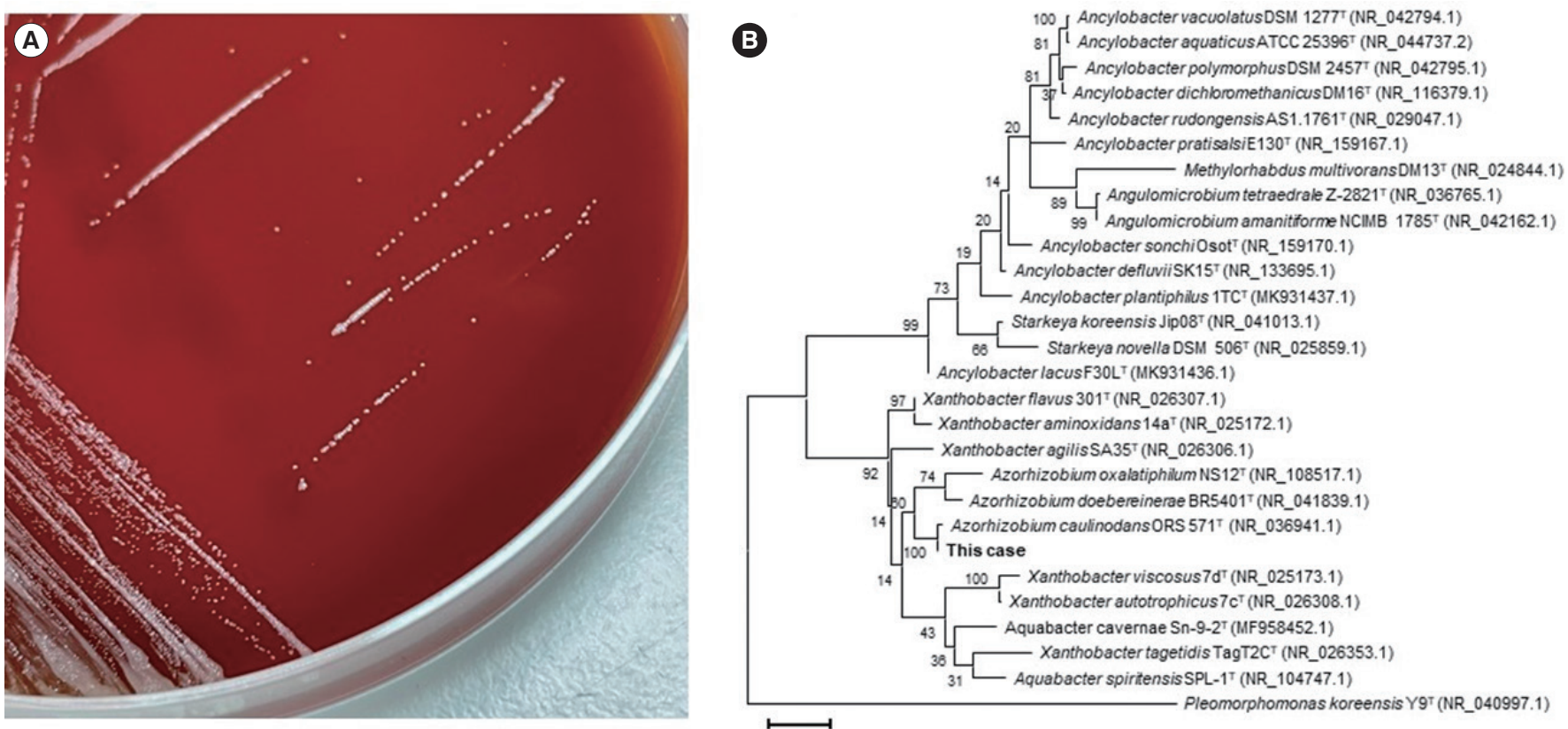

Fig. 1. Colony morphology of the Azorhizobium caulinodans isolate and a phylogenetic tree based on 16S rRNA gene sequences. (A) Round, cream-colored pinpoint colonies on a blood agar plate. (B) Phylogenetic tree constructed by the maximum-likelihood method using the model GTR +I+G and Pleomorphomonas koreensis Y9 (NR_040997.1) as the subgroup. Bootstrap values are expressed as percentages of 1,000 replications, and the scale bar indicates the estimated number of substitutions per base. GenBank accession numbers are indicated within parentheses.

Table 1. Antimicrobial susceptibility of the Azorhizobium caulinodans clinical blood-culture isolate as determined using Etest

\begin{tabular}{lcc}
\hline Antimicrobial agent & MIC $(\mu \mathrm{g} / \mathrm{mL})$ & Interpretation \\
\hline Piperacillin/tazobactam & $\geq 256 / 4$ & $\mathrm{R}$ \\
Cefotaxime & $\geq 32$ & $\mathrm{I} / \mathrm{R}$ \\
Ceftriaxone & $\geq 32$ & $\mathrm{I} / \mathrm{R}$ \\
Ceftazidime & $\geq 256$ & $\mathrm{R}$ \\
Imipenem & $\geq 32$ & $\mathrm{R}$ \\
Meropenem & $\geq 32$ & $\mathrm{R}$ \\
Gentamicin & 0.5 & $\mathrm{~S}$ \\
Ciprofloxacin & 1.5 & $\mathrm{I}$ \\
Levofloxacin & 1 & $\mathrm{~S}$ \\
Moxifloxacin & 0.19 & $\mathrm{NA}$ \\
Clindamycin & 16 & $\mathrm{NA}$ \\
Trimethoprim/sulfamethoxazole & $0.012 / 0.228$ & $\mathrm{~S}$
\end{tabular}

Abbreviations: MIC, minimal inhibitory concentration; $R$, resistant; I, intermediate; S, susceptible; NA, not available.

identified the isolate as A. caulinodans (Fig. 1B). Antimicrobial susceptibility tests (ASTs) were performed by measuring minimal inhibitory concentrations (MICs) using Etest (bioMérieux, Marcy l'Étoile, France). Etest strips were placed on MuellerHinton agar and read after 48 hours. AST results, interpreted according to other non-Enterobacterales per the CLSI guidelines, are presented in Table 1 [4].

On day 6, two pairs of CVCs and two pairs of peripheral blood follow-up blood cultures were prepared. A. caulinodans was detected in the two pairs of CVC blood cultures and confirmed using $16 \mathrm{~S}$ rRNA gene sequencing. On day 8, he developed neutropenia. On day 10, his CRP level decreased to $20.0 \mathrm{mg} / \mathrm{L}$, and the pleural effusion was drained by ultrasound-guided aspiration; however, no microorganisms were recovered from the pleural fluid culture. Follow-up CVC and peripheral blood cultures on days 10 and 15 were negative. After resolving the chemotherapy-caused neutropenia, he was discharged on day 20. Piperacillin/tazobactam was administered until discharge.

Azorhizobium spp. belong to rhizobia, a group of soil bacteria that form nodules on the roots of legumes [5]. Among them, the Burkholderia cepacia complex is a major human pathogen [5], and Agrobacterium (formerly Rhizobium) pusense infections are increasing [6].

During decitabine induction in elderly AML patients, neutropenic fever and infection are major adverse events [7]. Because the patient was previously admitted to a nursing hospital, there was no risk of soil exposure. He appeared to have developed the bacteremia owing to decreased immunity caused by AML. 
The isolate showed high MICs for $\beta$-lactam antibiotics and positive for $\beta$-lactamase on a Cefinase test (Becton Dickinson, Sparks, MD, USA), possibly attributable to a class A $\beta$-lactamase gene (penA) [8]. However, the bacteremia was resolved with piperacillin/tazobactam, to which the isolate was resistant. Identification and ASTs were conducted after discharge, and thus, the antibiotics could not be changed during hospitalization. The bacteremia probably improved due to an increase in neutrophils or in vivo piperacillin/tazobactam activity.

The isolate was misidentified by VITEK 2 and failed to be identified by MALDI-TOF MS. The identification of root nodule bacteria by MALDI-TOF MS has been reported [9]. By analyzing the mass-to-charge ratio of the isolate using Ribopeaks, the database of which includes environmental bacteria, the isolate was accurately identified as A. caulinodans [10]. Unusual bacteria may be accurately identified by $16 \mathrm{~S}$ rRNA sequencing and MALDI-TOF MS using a comprehensive environmental bacteria database.

\section{ACKNOWLEDGEMENTS}

None.

\section{AUTHOR CONTRIBUTIONS}

Research conception and design: Park JH. Data acquisition: Park JH, Kim TS, Park H. Data analysis and interpretation: Park $\mathrm{JH}$. Figure preparation: Park JH. Drafting of the manuscript: Park JH. Revision of the manuscript: Park JH, Kim TS, Park H. Approval of the final manuscript: all authors.

\section{CONFLICTS OF INTEREST}

No potential conflicts of interest relevant to this article are reported.

\section{RESEARCH FUNDING}

This work was supported by the Seoul National University Hospital Research Fund (grant number: 0420150750).

\section{ORCID}

Jae Hyeon Park https://orcid.org/0000-0003-0261-2185

Taek Soo Kim https://orcid.org/0000-0002-2093-1721

Hyunwoong Park https://orcid.org/0000-0001-7765-2259

\section{REFERENCES}

1. Dreyfus B, Garcia JL, Gillis M. Characterization of Azorhizobium caulinodans gen. nov., sp. nov., a stem-nodulating nitrogen-fixing bacterium isolated from Sesbania rostrata. Int J Syst Evol Microbiol 1988;38:8998.

2. Jiang N, Liu W, Li Y, Wu H, Zhang Z, Alexandre G, et al. A chemotaxis receptor modulates nodulation during the Azorhizobium caulinodansSesbania rostrata symbiosis. Appl Environ Microbiol 2016;82:3174-84.

3. CLSI. Interpretive criteria for identification of bacteria and fungi by targeted DNA sequencing. 2nd ed. MM18. Wayne, PA: Clinical and Laboratory Standards Institute, 2018.

4. CLSI. Performance standards for antimicrobial susceptibility testing. 30th ed. M100. Wayne, PA: Clinical and Laboratory Standards Institute, 2020.

5. Berrada $\mathrm{H}$ and Fikri-Benbrahim K. Taxonomy of the rhizobia: current perspectives. Brit Microbiol Res J 2014;4:616-39.

6. Aujoulat F, Marchandin H, Zorgniotti I, Masnou A, Jumas-Bilak E. Rhizobium pusense is the main human pathogen in the genus Agrobacterium/Rhizobium. Clin Microbiol Infect 2015;21:472.e1-5.

7. Short NJ, Kantarjian HM, Loghavi S, Huang X, Qiao W, Borthakur G, et al. Treatment with a 5-day versus a 10-day schedule of decitabine in older patients with newly diagnosed acute myeloid leukaemia: a randomised phase 2 trial. Lancet Haematol 2019;6:e29-e37.

8. Hirakawa T, Matsuoka J-I, Morohashi K, Hidaka M, Ogawa T, Aono T. LysR-type transcriptional regulator of high temperature inducible class A $\beta$-lactamase gene controls pathogenic R-body production genes in Azorhizobium caulinodans. Soil Sci Plant Nutr 2021;67:57-70.

9. Ziegler D, Pothier JF, Ardley J, Fossou RK, Pflüger V, de Meyer S, et al. Ribosomal protein biomarkers provide root nodule bacterial identification by MALDI-TOF MS. Appl Microbiol Biotechnol 2015;99:5547-62.

10. Tomachewski D, Galvão CW, de Campos Júnior A, Guimarães AM, Ferreira da Rocha JC, Etto RM. Ribopeaks: a web tool for bacterial classification through $\mathrm{m} / \mathrm{z}$ data from ribosomal proteins. Bioinformatics 2018; 34:3058-60. 